\title{
NEUTROSOPHIC FEEBLY NORMAL SPACES
}

\section{P. JEYA PUVANESWARI ${ }^{1}$, K. BAGEERATHI ${ }^{2}$ \& P. SELVAN ${ }^{2}$}

${ }^{1}$ Department of Mathematics, Vivekananda College, Agasteeswaram, Tamil Nadu, India

${ }^{2}$ Department of Mathematics, Aditanar College of Arts and Science, Tiruchendur, Tamil Nadu, India

\section{ABSTRACT}

In this section, we introduce neutrosophic feebly normal and strongly neutrosophic feebly normal spaces using neutrosophic feebly open set and neutrosophic feebly closed sets. Also, found their relations among themselves and with already existing spaces. Also, we discussed some basic properties and the characterizations of already mentioned spaces. AMS Subject Classification: $03 E 72$

KEYWORDS: Neutrosophic Feebly Closed Set, Neutrosophic Feebly Open Set \& Neutrosophic Feebly Normal and Strongly Neutrosophic Feebly Normal Spaces

Received: Apr 12, 2019; Accepted: May 02, 2019; Published: May 21, 2019; Paper Id.: IJCMSJUN201913

\section{INTRODUCTION}

Fuzzy sets were introduced by Zadeh [19] in 1965. The concepts of intuitionistic fuzzy sets by K. Atanassov [2], several researches were conducted on the generalizations of the notion of intuitionistic fuzzy sets. FloretinSmarandache [6-9] developed Neutrosophic set \&logic is a generalization of the Intuitionistic Fuzzy Logic\& set respectively. Neutrosophic technique is a special technique is based on Neutrosophy. This theory developed by“FlorentinSmarandache"in 1995. In Neutrosophy consider every notion or idea is together with A, Anti-AandNeut-A here Anti-A is the opposite or negation of A, Neut-A is the field of "neutralities". Neutrosophic method is derived fromFuzzy logic or in intuitionistic Fuzzy logic. The first bookwas published on Neutrosophy as a title of book is Neutrosophy probability, set and logic in 1998. The word Neutrosophy introduced from Latin "neuter" - neutral, Greek "Sophia"- skill/wisdom.Neutrosophy means sill on neutrals. The main task of this study is to apply neutrosophic method to the generaltheory of relativity, aiming to discover new hidden effects. Here it's why we decided to employ neutrosophic method in this field. Neutrosophic method means to find common features to uncommon entities. T, I,Farecalled neutrosophic components will represent the truth value, indeterminacy value and false hood valuerespectively referring to neutrosophic methods. A. A. Salama\& S. A. Alblowi [1] introduced and studied Neutrosophic Topological spaces and its continuous function in [17]. Iswarya et al. [10] defined the concept of neutrosophic semi open sets in neutrosophic topological spaces. JeyaPuvaneswari et al. [12-14] defined neutrosophic feebly open sets, neutrosophic feebly closed sets and neutrosophic feebly continuous functions in neutrosophic topological spaces.

In this paper, the concept of neutrosophic feebly normalin neutrosophic topological spaces is introduced. Further, the work is extended as strongly neutrosophic feebly normal in neutrosophic topological spaces and establishes some of their related attributes. 
This paper is organized as follows. Section II gives the basic definitions of neutrosophic feebly open sets\& closed sets, neutrosophic feebly continuous functions and neutrosophic feebly compactin neutrosophic topological spaces and their properties which are used in the later sections. The Section III deals with the concept of neutrosophic feebly normal space. Section IV explains strongly neutrosophic feebly normalin neutrosophic topological spaces and their properties.

\section{PRELIMINARIES}

Here in this paper the neutrosophic topological space is denoted by $(X, \tau)$. Also the neutrosophic interior, neutrosophic closure of a neutrosophic set $\mathrm{A}$ are denoted by $\mathrm{NInt}(\mathrm{A})$ and $\mathrm{NCl}(\mathrm{A})$. The complement of a neutrosophic set $\mathrm{A}$ is denoted by $\mathrm{A}^{\mathrm{C}}$ and the empty and whole sets are denoted by $0_{\mathrm{N}}$ and $1_{\mathrm{N}}$ respectively.

Definition 2.1. [15] Let $X$ be a non-empty fixed set. A neutrosophic set $A$ is an object having the form $\mathrm{A}=\{\langle\mathrm{x}$, $\left.\left.\mu_{\mathrm{A}}(\mathrm{x}), \sigma_{\mathrm{A}}(\mathrm{x}), \gamma_{\mathrm{A}}(\mathrm{x})\right\rangle: \mathrm{x} \in \mathrm{X}\right\}$ where $\mu_{\mathrm{A}}(\mathrm{x}), \sigma_{A}(x)$ and $\gamma_{A}(x)$ which represents the degree of membership function, the degree indeterminacy and the degree of non-membership function respectively of each element $x \in X$ to the set $A$.

Neutrosophic sets $0_{N}$ and $1_{N}$ in $X$ as follows:

$0_{N}$ may be defined as:

$\left(0_{1}\right) 0_{N}=\{\langle x, 0,0,1\rangle: x \in X\}$

$\left(0_{2}\right) 0_{N}=\{\langle x, 0,1,1\rangle: x \in X\}$

$\left(0_{3}\right) 0_{N}=\{\langle x, 0,1,0\rangle: x \in X\}$

$\left(0_{4}\right) 0_{N}=\{\langle x, 0,0,0\rangle: x \in X\}$

$1_{N}$ may be defined as:

$\left(1_{1}\right) 1_{N}=\{\langle x, 1,0,0\rangle: x \in X\}$

$\left(1_{2}\right) 1_{N}=\{\langle x, 1,0,1\rangle: x \in X\}$

$\left(1_{3}\right) 1_{N}=\{\langle x, 1,1,0\rangle: x \in X\}$

$\left(1_{4}\right) 1_{N}=\{\langle x, 1,1,1\rangle: x \in X\}$

Definition 2.2. [15] Let $A=\left\langle\mu_{A}, \sigma_{A}, \gamma_{A}\right\rangle$ be a neutrosophic set in $X$. Then the complement of the set $A\left[A^{C}\right.$ for short] may be defined as three kinds of complements:

$\left(\mathrm{C}_{1}\right) A^{C}=\left\{\left\langle x, 1-\mu_{A}(x), 1-\sigma_{A}(x), 1-\gamma_{A}(x)\right\rangle: x \in X\right\}$

$\left(\mathrm{C}_{2}\right) A^{C}=\left\{\left\langle x, \gamma_{A}(x), \sigma_{A}(x), \mu_{A}(x)\right\rangle: x \in X\right\}$

$\left(\mathrm{C}_{3}\right) A^{C}=\left\{\left\langle x, \gamma_{A}(x), 1-\sigma_{A}(x), \mu_{A}(x)\right\rangle: x \in X\right\}$

One can define several relations and operations between neutrosophic setsfollow:

Definition 2.3. [15] Let $x$ be a non-empty set, and neutrosophic sets $A$ and $B$ in the form $A=\left\{\left\langle x, \mu_{A}(x), \sigma_{A}(x), \gamma_{A}(x)\right.\right.$ \rangle$: x \in X\}$ and $B=\left\{\left\langle x, \mu_{B}(x), \sigma_{B}(x), \gamma_{B}(x)\right\rangle: x \in X\right\}$. Then we may consider two possible definitions for subsets $(A \leq B)$.

$A \leq B$ may be defined as: 
(1) $A \leq B \Leftrightarrow \mu_{A}(x) \leq \mu_{B}(x), \sigma_{A}(x) \leq \sigma_{B}(x)$ and $\gamma_{A}(x) \geq \gamma_{B}(x), \forall x \in X$

(2) $A \leq B \Leftrightarrow \mu_{A}(x) \leq \mu_{B}(x), \sigma_{A}(x) \geq \sigma_{B}(x)$ and $\gamma_{A}(x) \geq \gamma_{B}(x), \forall x \in X$

Definition 2.4. [15] Let $X$ be a non-empty set, and $A=\left\langle x, \mu_{A}(x), \sigma_{A}(x), \gamma_{A}(x)\right\rangle, B=\left\langle x, \mu_{B}(x), \sigma_{B}(x), \gamma_{B}(x)\right\rangle$ are neutrosophic subsets. Then

(1) $A \wedge B$ may be defined as:

$\left(\mathrm{I}_{1}\right) \mathrm{A} \wedge \mathrm{B}=\left\langle\mathrm{x}, \mu_{\mathrm{A}}(\mathrm{x}) \wedge \mu_{\mathrm{B}}(\mathrm{x}), \sigma_{\mathrm{A}}(\mathrm{x}) \wedge \sigma_{\mathrm{B}}(\mathrm{x})\right.$ and $\left.\gamma_{\mathrm{A}}(\mathrm{x}) \vee \gamma_{\mathrm{B}}(\mathrm{x})\right\rangle$

$\left(\mathrm{I}_{2}\right) \mathrm{A} \wedge \mathrm{B}=\left\langle\mathrm{x}, \mu_{\mathrm{A}}(\mathrm{x}) \wedge \mu_{\mathrm{B}}(\mathrm{x}), \sigma_{\mathrm{A}}(\mathrm{x}) \vee \sigma_{\mathrm{B}}(\mathrm{x})\right.$ and $\left.\gamma_{\mathrm{A}}(\mathrm{x}) \vee \gamma_{\mathrm{B}}(\mathrm{x})\right\rangle$

(2) AVB may be defined as:

$\left(\mathrm{U}_{1}\right) \mathrm{A} \vee \mathrm{B}=\left\langle\mathrm{x}, \mu_{\mathrm{A}}(\mathrm{x}) \vee \mu_{\mathrm{B}}(\mathrm{x}), \sigma_{\mathrm{A}}(\mathrm{x}) \vee \sigma_{\mathrm{B}}(\mathrm{x})\right.$ and $\left.\gamma_{\mathrm{A}}(\mathrm{x}) \wedge \gamma_{\mathrm{B}}(\mathrm{x})\right\rangle$

$\left(\mathrm{U}_{2}\right) \mathrm{A} \vee \mathrm{B}=\left\langle\mathrm{x}, \mu_{\mathrm{A}}(\mathrm{x}) \vee \mu_{\mathrm{B}}(\mathrm{x}), \sigma_{\mathrm{A}}(\mathrm{x}) \wedge \sigma_{\mathrm{B}}(\mathrm{x})\right.$ and $\left.\gamma_{\mathrm{A}}(\mathrm{x}) \wedge \gamma_{\mathrm{B}}(\mathrm{x})\right\rangle$

Definition 2.5. [15] A neutrosophic topology [NT for short] is a non-empty set $X$ is a family $\tau$ of neutrosophic subsets in $X$ satisfying the following axioms:

$\left(\mathrm{NT}_{1}\right) 0_{N}, 1_{N} \in \tau$

$\left(\mathrm{NT}_{2}\right) G_{1} \wedge G_{2} \in \tau$ for any $G_{1}, G_{2} \in \tau$,

$\left(\mathrm{NT}_{3}\right) \bigvee G_{i} \in \tau$ for every $\left\{G_{i}: \mathrm{i} \in \mathrm{J}\right\} \leq \tau$

In this case the pair $(X, \tau)$ is called a neutrosophic topological space. The elements of $\tau$ are called neutrosophic open sets.

Definition 2.6. [15] The complement of $A$ [ $A^{C}$ for short] of neutrosophic open sets are called neutrosophic closed set in $X$.

Definition 2.7[15]Let $\mathrm{X}$ and $\mathrm{Y}$ be two nonempty neutrosophic sets and $\mathrm{f}: \mathrm{X} \rightarrow \mathrm{Y}$ be a function.

(i) If $\mathrm{B}=\left\{\left(\mathrm{y}, \mu_{\mathrm{B}}(\mathrm{y}), \sigma_{\mathrm{B}}(\mathrm{y}), \gamma_{\mathrm{B}}(\mathrm{y})\right): \mathrm{y} \in \mathrm{Y}\right\}$ is a Neutrosophic set in $\mathrm{Y}$, then the pre image of $\mathrm{B}$ under $\mathrm{f}$ is denoted and defined by $f^{1}(B)=\left\{\left(x, f^{-1}\left(\mu_{B}\right)(x), f^{-1}\left(\sigma_{B}\right)(x), f^{-1}\left(\gamma_{B}\right)(x)\right): x \in X\right\}$.

(ii) If $\left.\mathrm{A}=\left\{<\mathrm{x}, \alpha_{\mathrm{A}}(\mathrm{x}), \delta_{\mathrm{A}}(\mathrm{x}), \lambda_{\mathrm{A}}(\mathrm{x})\right): \mathrm{x} \in \mathrm{X}\right\}$ is a NS in $\mathrm{X}$, then the image of $\mathrm{A}$ under $\mathrm{f}$ is denoted and definedby $f(A)=\left\{\left(y, f\left(\alpha_{A}\right)(y), f\left(\delta_{A}\right)(y), f_{-}\left(\lambda_{A}\right)(y)\right): y \in Y \quad\right\}$ where $f_{-}\left(\lambda_{A}\right)=C(f(C(A)))$.

In (i), (ii), since $\mu_{B}, \sigma_{B}, \gamma_{B}, \alpha_{A}, \delta_{A}, \lambda_{A}$ are neutrosophic sets, we explain that $f^{-1}\left(\mu_{B}\right)(x)=\mu_{B}(f(x))$,

andf $\left(\alpha_{\mathrm{A}}\right)(\mathrm{y})== \begin{cases}\sup \left\{\alpha_{\mathrm{A}}(\mathrm{x}): \mathrm{x} \in \mathrm{f}^{-1}(\mathrm{y})\right\}, & \text { if } \mathrm{f}^{-1}(\mathrm{y}) \neq \varphi \\ 0 & \text { otherwise }\end{cases}$

Lemma 2.8. [16]Let $f: X \rightarrow Y$ be a function. If $A$ is a neutrosophic subset of $\mathrm{X}$ and $\mu$ is a neutrosophic subset of Y. Then

(i) $\quad f\left(f^{-1}(A)\right) \leq A$ 
(ii) $\quad f\left(f^{-1}(A)\right)=A \Leftrightarrow f$ is surjective.

$($ iii $) f^{-1}(f(A)) \geq A$

(iv) $f^{-1}(f(A))=A$ whenever $f$ is injective.

Definition 2.9. [12]A neutrosophic subset $A$ of a neutrosophic topological space $(X, \tau)$ is neutrosophic feebly open if there is a neutrosophic open set $U$ in $X$ such that $U \leq A \leq N \operatorname{Scl}(U)$.

Lemma 2.10. [12] (i) Every neutrosophic open set is a neutrosophic feebly open set.

Lemma 2.11. [12] A neutrosophic subset $A$ of a neutrosophic topological space $(X, \tau)$ is neutrosophic feebly closed if and only if $\operatorname{Ncl}(\operatorname{Nint}(\operatorname{Ncl}(A))) \leq A$.

Definition 2.11. [15] Let $(X, \tau)$ and $(Y, \sigma)$ be neutrosophic topological spaces. Then a map $f:(X, \tau) \rightarrow(Y, \sigma)$ is called neutrosophic continuous (in short N-continuous) function if the inverse image of every neutrosophic open set in $(Y, \sigma)$ is neutrosophic open set in $(X, \tau)$.

Definition 2.12.[14]Let $(X, \tau)$ and $(Y, \sigma)$ be two neutrosophic topological spaces. A function $f: X \rightarrow Y$ is called neutrosophic feebly irresolute if the inverse image of every neutrosophic feebly open set in $Y$ is neutrosophic feebly open in $X$.

Lemma2.13.[14]Let $(X, \tau)$ and $(Y, \sigma)$ be two neutrosophic topological spaces. A function $f: X \rightarrow Y$ is neutrosophic feebly irresolute if and only if the inverse image of every neutrosophic feebly closed set in $Y$ is neutrosophic feebly closed in $X$.

Lemma2.14.[14]Everyneutrosophic feebly irresolute function is neutrosophic feebly continuous.

Definition $2.15[12]$ Let $\alpha, \beta, \gamma \in[0,1]$ and $\alpha+\beta+\gamma \leq 1$. A Neutrosophic point with support $x_{(\alpha, \beta, \gamma)} \in \mathrm{X}$ is a neutrosophic set of $X$ is defined by $x_{(\alpha, \beta, \gamma)}=\left\{\begin{array}{l}(\alpha, \beta, \gamma), y=x \\ (0,0,1), y \neq x\end{array}\right.$

In this case, $\mathrm{x}$ is called the support of $\mathrm{x}_{(\alpha, \beta, \gamma)}$ and $\alpha, \beta$ and $\gamma$ are called the value, intermediate value and the non value of $x_{(\alpha, \beta, \gamma)}$ respectively. A Neutrosophic point $x_{(\alpha, \beta, \gamma)}$ is said to belong to a neutrosophic set $A=\left\{\left\langle x, \mu_{A}(x), \sigma_{A}(x)\right.\right.$, $\left.\left.\gamma_{A}(x)\right\rangle: x \in X\right\}$ is denoted by two ways(i) $x_{(\alpha, \beta, \gamma)} \in A$ if $\alpha \leq \mu_{A}(x), \beta \leq \sigma_{A}(x)$ and $\gamma \geq \gamma_{A}(x)$.

(ii) $x_{(\alpha, \beta, \gamma)} \in A$ if $\alpha \leq \mu_{A}(x), \beta \geq \sigma_{A}(x)$ and $\gamma \geq \gamma_{A}(x)$.

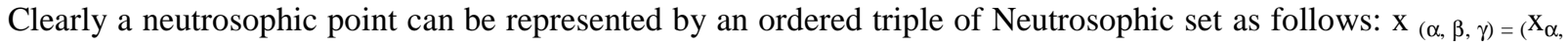
$\left.\mathrm{x}_{\beta}, \mathrm{C}\left(\mathrm{x}_{\mathrm{c}(\gamma)}\right)\right)$. A class of all neutrosophic points in $\mathrm{X}$ is denoted as $\mathrm{NP}(\mathrm{X})$.

Definition 2.16.[13]Let $(X, \tau)$ and $(Y, \sigma)$ be two neutrosophic topological spaces. Then a function $f: X \rightarrow Y$ is said to be neutrosophic feebly continuous function if $f^{-1}(G)$ is neutrosophic feebly open set in X for all neutrosophic open set $G$ in $Y$.

Definition 2.17.[14] Let $(X, \tau)$ and $(Y, \sigma)$ be two neutrosophic topological spaces. A function $f: X \rightarrow Y$ is said to be neutrosophic feebly closed if the image of each neutrosophicclosed set in $X$ is neutrosophic feebly closed in $Y$. 
Definition 2.18. [14]Let $(X, \tau)$ and $(Y, \sigma)$ be two neutrosophic topological spaces. A function $f: X \rightarrow Y$ is said to be neutrosophic feebly open if the image of each neutrosophicopen set in $X$ is neutrosophic feebly open in $Y$.

Theorem 2.19. [14]For any bijective map $f: X \rightarrow Y$ the following statements are equivalent:

(i) $f^{-1}: Y \rightarrow X$ is neutrosophic feebly continuous.

(ii) $f$ isneutrosophic feebly open.

(iii) $\quad f$ isneutrosophic feebly closed.

Definition 2.20.[3] A collection $\mathcal{B}$ of neutrosophic feebly open sets in $X$ is called a neutrosophic feebly open cover of a subset $B$ of $X$ if $B \leq \bigvee\left\{U_{\alpha}: U_{\alpha} \in \mathcal{B}\right\}$.

Definition 2.21.[3] A topological space $X$ is said to be neutrosophic feebly compact if every neutrosophic feebly open cover of $X$ has a finite subcover.

\section{NEUTROSOPHIC FEEBLY NORMAL SPACES}

In this section, we introduceneutrosophic feebly normal spaces and study their properties.

Definition 3.1. A neutrosophic topological space $(X, \tau)$ is said to be neutrosophic feebly normal if for any two disjoint neutrosophic feebly closed sets $A$ and $B$, there exist disjoint neutrosophic feebly open sets $U$ and $V$ such that $A \leq U$ and $B \leq V$.

Theorem 3.2. In a neutrosophic topological space $X$, the following are equivalent:

- $\quad X$ is neutrosophic feebly normal.

- For every neutrosophic feebly closed set $A$ in $X$ and every neutrosophic feebly open set $U$ containing $A$, there exists a neutrosophic feebly open set $V$ containing $A$ such that $N F c l(V) \leq U$.

- $\quad$ For each pair of disjoint neutrosophic feebly closed sets $A$ and $B$ in $X$, there exists a neutrosophic feebly open set $U$ containing $A$ such that $\operatorname{NFcl}(U) \wedge B=0_{N}$.

- $\quad$ For each pair of disjoint neutrosophic feebly closed sets $A$ and $B$ in $X$, there exist neutrosophic feebly open sets $U$ and $V$ containing $A$ and $B$ respectively such that $N F c l(U) \wedge N F c l(V)=0_{N}$.

\section{Proof}

(a) $\rightarrow$ (b): Let $U$ be a neutrosophic feebly open set containing the neutrosophic feebly closed set $A$. Then $B=U^{C}$ is a neutrosophic feebly closed set disjoint from $A$. Since $X$ is neutrosophic feebly normal, there exist disjoint neutrosophic feebly open sets $V$ and $W$ containing $A$ and $B$ respectively. Then $\operatorname{NFcl}(V)$ is disjoint from $B$.Since if $y_{(r, t, s)} \in B$, the set $W$ is a neutrosophic feebly open set containing $y_{(r, t, s)}$ disjoint from $V$. Hence $\operatorname{NFcl}(V) \leq U$.

(b) $\rightarrow$ (c): Let $A$ and $B$ be disjoint neutrosophic feebly closed sets in $X$. Then $B^{C}$ is a neutrosophic feebly open set containing $A$. By (b), there exists a neutrosophic feebly open set $U$ containing $A$ such that $N F c l(U) \leq B^{C}$. Hence $\operatorname{NFcl}(U) \wedge B=0_{N}$. This proves (c).

(c) $\rightarrow(\mathrm{d})$ : Let $A$ and $B$ be disjoint neutrosophic feebly closed sets in $X$. Then, by (c), there exists a neutrosophic 
feebly open set $U$ containing $A$ such that $N F c l(U) \wedge B=0_{N}$. Since $N F c l(U)$ is neutrosophic feebly closed, $B$ and $N F c l(U)$ are disjoint neutrosophic feebly closed sets in $X$. Again by (c), there exists a neutrosophic feebly open set $V$ containing $B$ such that $N F c l(U) \wedge N F c l(V)=0_{N}$.This proves (d).

(d) $\rightarrow(a)$ : Let $A$ and B be the disjoint neutrosophic feebly closed sets in X. By (d), there exist neutrosophic feebly open sets $\mathrm{U}$ and $\mathrm{V}$ containing $\mathrm{A}$ and B respectively such that $N F c l(U) \wedge N F c l(V)=0_{N}$. Since $U \wedge V \leq N F c l(U) \wedge N F c l(V)$, $U$ and $V$ are disjoint neutrosophic feebly open sets containing $A$ and $B$ respectively. Thus $X$ is neutrosophic feebly normal.

Theorem 3. 3. A neutrosophic topological space $(X, \tau)$ is neutrosophic feebly normal if and only if for every neutrosophic feebly closed set $F$ and neutrosophic feebly open set $G$ containing $F$, there exists a neutrosophic feebly open set $V$ such that $F \leq V \leq N F c l(V) \leq G$.

\section{Proof}

Let $(X, \tau)$ be neutrosophic feebly normal. Let $F$ be a neutrosophic feebly closed set and let $G$ be a neutrosophic feebly open set containing $F$. Then $F$ and $G^{c}$ are disjoint neutrosophic feebly closed sets. Since $X$ is neutrosophic feebly normal, there exist disjoint neutrosophic feebly open sets $V_{1}$ and $V_{2}$ such that $F \leq V_{1}$ and $G^{C} \leq V_{2}$. Thus $F \leq V_{1} \leq V_{2}{ }^{C} \leq$ $G$. Since $V_{2}{ }^{C}$ is neutrosophic feebly closed, so $\operatorname{NFcl}\left(\mathrm{V}_{1}\right) \leq N F c l\left(V_{2}{ }^{C}\right)=V_{2}{ }^{C} \leq G$. Take $V=V_{1}$. This implies that $F \leq V \leq$ $\operatorname{NFcl}(V) \leq G$.

Conversely, suppose the condition holds. Let $H_{1}$ and $H_{2}$ be two disjoint neutrosophic feebly closed sets in $X$. Then $\mathrm{H}_{2}{ }^{C}$ is a neutrosophic feebly open set containing $H_{1}$. By assumption, there exists a neutrosophic feebly open set $V$ such that $H_{1} \leq V \leq N F c l(V) \leq H_{2}{ }^{C}$. Since $V$ is neutrosophic feebly open and $N F c l(V)$ is neutrosophic feebly closed. Then $(\operatorname{NFcl}(V))^{C}$ is neutrosophic feebly open. Now $N F c l(V) \leq H_{2}{ }^{C}$ implies that $H_{2} \leq(\operatorname{NFcl}(V))^{C}$. AlsoV $\Lambda(N F c l(V))^{C} \leq$ $N F c l(V) \wedge(N F c l(V))^{C}=0_{N}$. That is $V$ and $(\operatorname{NFcl}(V))^{C}$ are disjoint neutrosophic feebly open sets containing $H_{1}$ and $H_{2}$ respectively. This shows that $(X, \tau)$ is neutrosophic feebly normal.

Theorem 3.4.For a space $X$, then the following are equivalent:

- $\quad$ is neutrosophic feebly normal.

- For any two neutrosophic feebly open sets $U$ and $V$ whose union is $1_{N}$, there exist neutrosophic feebly closed subsets $A$ of $U$ and $B$ of $V$ whose union is also $X$.

\section{Proof}

(a) $\rightarrow$ (b): Let $U$ and $V$ be two neutrosophic feebly open sets in a neutrosophic feebly normal space $X$ such that $1_{N}=U \vee V$. Then $U^{C}, V^{C}$ are disjoint neutrosophic feebly closed sets. Since $X$ is neutrosophic feebly normal, then there exist disjoint neutrosophic feebly open sets $G_{1}$ and $G_{2}$ such that $U^{C} \leq G_{1}$ and $V^{C} \leq G_{2}$. Let $A=G_{1}{ }^{C}$ and $=G_{2}{ }^{C}$. Then $A$ and $B$ are neutrosophic feebly closed subsets of $U$ and $V$ respectively such that $A \bigvee B=1_{N}$. This proves (b).

(b) $\rightarrow$ (a): Let $A$ and $B$ be disjoint neutrosophic feebly closed sets in $X$. Then $A^{C}$ and $B^{C}$ are neutrosophic feebly open sets whose union is $1_{N}$. By (b), there exists neutrosophic feebly closed sets $F_{1}$ and $F_{2}$ such that $F_{1} \leq A^{C}, \mathrm{~F}_{2} \leq B^{C}$ and $F_{1} \vee F_{2}=1_{N}$. Then $F_{1}{ }^{C}$ and $F_{2}{ }^{C}$ are disjoint neutrosophic feebly open sets containing $A$ and $B$ respectively. Therefore $X$ is neutrosophic feebly normal. 
Theorem 3. 5. Let $f:(X, \tau) \rightarrow(Y, \tau)$ be a function.

- If $f$ is injective, neutrosophic feebly irresolute, neutrosophic feebly open and $X$ is neutrosophic feebly normal then $Y$ is neutrosophic feebly normal.

- If $f$ is neutrosophic feebly irresolute, neutrosophic feebly closed and $Y$ is neutrosophic feebly normal then $X$ is neutrosophic feebly normal.

\section{Proof}

(a) Suppose $X$ is neutrosophic feebly normal. Let $A$ and $B$ be disjoint neutrosophic feebly closed sets in $Y$. Since $f$ is neutrosophic feebly irresolute, $f^{-1}(A)$ and $f^{-1}(B)$ are neutrosophic feebly closed in $X$. Since $X$ is neutrosophic feebly normal, there exist disjoint neutrosophic feebly open sets $U$ and $V$ in $\mathrm{X}$ such that $f^{-1}(A) \leq U$ and $f^{-1}(B) \leq V$. Now $f^{-1}(\mathrm{~A}) \leq U \Rightarrow A \leq f(U)$ and $f^{-1}(B) \leq V \Rightarrow B \leq f(V)$. Since $f$ is a neutrosophic feebly open map, $f(U)$ and $f(V)$ are neutrosophic feebly open in $Y$. Also $U \wedge V=0_{N} \Rightarrow f(U \wedge V)=0_{N}$ and $f$ is injective, then $f(U) \wedge f(V)=0_{N}$. Thus $f(U)$ and $f(V)$ are disjoint neutrosophic feebly open sets in $Y$ containing $A$ and $B$ respectively. Thus, $Y$ is neutrosophic feebly normal.

(b)Suppose $Y$ is neutrosophic feebly normal. Let $A$ and $B$ be disjoint neutrosophic feebly closed sets in $X$. Since $f$ is neutrosophic feeblyirresolute and neutrosophic feebly closed, $f(A)$ and $f(B)$ are neutrosophic feebly closed in $Y$. Since $Y$ is neutrosophic feebly normal, there exist disjoint neutrosophic feebly open sets $U$ and $V$ in $Y$ such that $f(A) \leq U$ and $f(B) \leq V$. That is $A \leq f^{-1}(U)$ and $B \leq f^{-1}(\mathrm{~V})$. Since $f$ is neutrosophic feeblyirresolute, $f^{-1}(U)$ and $f^{-1}(V)$ are disjoint neutrosophic feebly open such that $A \leq f^{-1}(U)$ and $B \leq f^{-1}(V)$. Thus $X$ is neutrosophic feebly normal.

Theorem 3.6. If given a pair of disjoint neutrosophic feebly closed sets $A, B$ of $X$, there is neutrosophic feebly continuous function $f$ such that $f(A)=0_{N}$ and $f(B)=1_{N}$, then $(X, \tau)$ is neutrosophic feebly normal.

\section{Proof}

Let $(X, \tau)$ be aneutrosophic topological space. Suppose for any pair of disjoint neutrosophic feebly closed sets A, $B$ in $X$, there exists a neutrosophic feebly continuous map $f$ such that $f(A)=0_{N}$ and $f(B)=1_{N}$. Let $E$ and $F$ be disjoint neutrosophic feebly closed sets in $\mathrm{X}$. LetG and Hbe disjoint neutrosophic feebly open sets. Since $\mathrm{f}$ is neutrosophic feeblycontinuous, $f^{-1}(G)$ and $f^{-1}(H)$ are neutrosophic feebly open in $X$. By our assumption, $f(E)=0_{N}$ and $f(F)=1_{N}$. Now $f(E)=0_{N}$ implies $f^{-1}(f(E)) \leq f^{-1}\left(0_{N}\right) \Rightarrow E \leq f^{-1}(f(E)) \leq f^{-1}\left(0_{N}\right) \Rightarrow E \leq f^{-1}\left(0_{N}\right)$. Similarly $F \leq f^{-1}\left(1_{N}\right)$. This implies that $E \leq f^{-1}\left(0_{N}\right) \leq f^{-1}(G)$. Then $F \leq f^{-1}\left(1_{N}\right) \leq f^{-1}(H)$. Further, $f^{-1}(G) \square f^{-1}(H)=f^{-1}(G \wedge H)=f^{-1}\left(0_{N}\right)=0_{N}$. So, we have a pair of disjoint neutrosophic feebly open sets, $f^{-1}(G), f^{-1}(H) \leq 1_{N}$ such that $E \leq f^{-1}(G)$ and $F \leq f^{-1}(H)$. This proves that $(X, \tau)$ is neutrosophic feebly normal

Theorem 3.7. If $f$ is a neutrosophic continuous, neutrosophic feebly open bijection of a neutrosophic normal space $X$ into a space $Y$ and if every neutrosophic feebly closed set in $Y$ is neutrosophic closed, then $Y$ is neutrosophic feebly regular. 


\section{Proof}

Let A and B be neutrosophic feebly closed setsin Y. Then by assumption, B is neutrosophic closed in Y. Since fis a neutrosophic continuous bijection, $\mathrm{f}^{-1}(\mathrm{~A})$ and $\mathrm{f}^{-1}(\mathrm{~B})$ is a neutrosophic closed set in $\mathrm{X}$. Since $\mathrm{X}$ is neutrosophic normal, there exist disjoint neutrosophic open sets $\mathrm{U} 1$ and $\mathrm{U} 2$ in $\mathrm{X}$ such that $\mathrm{f}^{-1}(\mathrm{~A}) \leq \mathrm{U} 1$ and $\mathrm{f}^{-1}(\mathrm{~B}) \leq \mathrm{U} 2$. Since fis neutrosophic feebly open, $\mathrm{f}(\mathrm{U} 1)$ and $\mathrm{f}(\mathrm{U} 2)$ are disjoint neutrosophic feebly open sets in Y containing A and B respectively. Hence Y is neutrosophic feebly normal.

\section{STRONGLY NEUTROSOPHIC FEEBLY NORMAL SPACES}

In this section, we introduce strongly neutrosophic feebly normal spaces and study their properties.

Definition 4.1. Aneutrosophic topological space $\mathrm{X}$ is said to be strongly neutrosophic feebly normal if for every pair of disjoint neutrosophic closed sets $A$ and $B$ in $X$, there are disjoint neutrosophic feebly open sets $U$ and $V$ in $X$ containing A and B respectively.

Theorem 4.2.Every neutrosophic feebly normal space is strongly neutrosophic feebly normal.

\section{Proof}

Suppose X is neutrosophic feebly normal. Let A and B be disjoint neutrosophic closed sets in X. Then A and B are neutrosophic feebly closed in X. Since X is neutrosophic feebly normal, there exist disjoint neutrosophic open sets $\mathrm{U}$ and $\mathrm{V}$ containing A and B respectively. Then by Proposition 2.1.16, $\mathrm{U}$ and $\mathrm{V}$ are neutrosophic feebly open in X.This implies that X is strongly neutrosophic feebly normal.

Theorem 4.3. In a neutrosophic topological space $\square$, the following are equivalent:

- Xis strongly neutrosophic feebly normal.

- For every neutrosophic closed set $\mathrm{F}$ in $\mathrm{X}$ and every neutrosophic open set $\mathrm{U}$ containing $\mathrm{F}$, there exists a neutrosophic feebly open set $\mathrm{V}$ containing F such that $\mathrm{NFcl}(\mathrm{V}) \leq \mathrm{U}$.

- For each pair of disjoint neutrosophic closed sets A and Bin X, there exists a neutrosophic feebly open set U containing A such that $\mathrm{NFcl}(\mathrm{U}) \wedge \mathrm{B}=0_{\mathrm{N}}$.

\section{Proof}

$(a) \rightarrow(b)$ : Let $U$ be a neutrosophic open set containing the neutrosophic closed set $F$. Then $H=U^{C}$ is a neutrosophic closed set disjoint from F. Since X is strongly neutrosophic feebly normal, there exist disjoint neutrosophic feebly open sets $\mathrm{V}$ and $\mathrm{W}$ containing $\mathrm{F}$ and $\mathrm{H}$ respectively. Then $\mathrm{NFcl}(\mathrm{V})$ is disjoint from $\mathrm{H}$, since if $\mathrm{y}_{(\mathrm{r}, \mathrm{t}, \mathrm{s})} \in \mathrm{H}$, the set $\mathrm{W}$ is a neutrosophic feebly open set containing $\mathrm{y}_{(\mathrm{r}, \mathrm{t}, \mathrm{s})}$ disjoint from $\mathrm{V}$. Hence $\mathrm{NFcl}(\mathrm{V}) \leq \mathrm{U}$.

$(\mathrm{b}) \rightarrow(\mathrm{c})$ : Let $\mathrm{A}$ and $\mathrm{B}$ be disjoint neutrosophic closed sets in $\mathrm{X}$. Then $\mathrm{B}^{\mathrm{C}}$ is a neutrosophic open set containing $\mathrm{A}$. By (b), there exists a neutrosophic feebly open set $U$ containing $A$ such that $\operatorname{NFcl}(U) \leq B^{C}$. $\operatorname{Hence} N F c l(U) \wedge B=0_{N}$. This proves (c).

$(\mathrm{c}) \rightarrow(\mathrm{a})$ : Let $\mathrm{A}$ and $\mathrm{B}$ be the disjoint neutrosophic feebly closed sets in X. By (c), there exists a neutrosophic feebly open set $U$ containing A such that $\operatorname{NFcl}(U) \wedge B=0_{N}$. Take $V=N F c l(U){ }^{C}$. Then $U$ and $V$ are disjoint neutrosophic feebly open sets containing A and B respectively. Thus X is strongly neutrosophic feebly normal. 
Theorem 4.4.For a neutrosophictopological space $\square$, then the following are equivalent:

- Xis strongly neutrosophic feebly normal.

- For any two neutrosophic open sets $\mathrm{U}$ and $\mathrm{V}$ whose union is $1_{\mathrm{N}}$, there exist neutrosophic feebly closed subsets $\mathrm{A}$ of $\mathrm{U}$ and $\mathrm{B}$ of $\mathrm{V}$ whose union is also $1_{\mathrm{N}}$.

\section{Proof}

(a) $\rightarrow$ (b): Let $\mathrm{U}$ and $\mathrm{V}$ be two neutrosophic open sets in a strongly neutrosophic feebly normal space $\mathrm{X}$ such that $1_{N}=U V V$. Then $U^{C}, V^{C}$ are disjointneutrosophic closed sets. Since $X$ is strongly neutrosophic feebly normal, then there exist disjoint neutrosophic feebly open sets $\mathrm{G} 1$ and $\mathrm{G} 2$ such that $\mathrm{U}^{\mathrm{C}} \leq \mathrm{G} 1$ and $\mathrm{V}^{\mathrm{C}} \leq \mathrm{G} 2$. Let $\mathrm{A}=\mathrm{G}_{1}{ }^{\mathrm{C}}$ and $\mathrm{B}=\mathrm{G}_{2}{ }^{\mathrm{C}}$. Then $\mathrm{A}$ and $\mathrm{B}$ are neutrosophic feebly closed subsets of $U$ and $V$ respectively such that $A \bigvee B=1_{N}$. This proves (b).

(b) $\rightarrow(a)$ : Let $A$ and $B$ be disjoint neutrosophic closed sets in $X$. Then $\mathrm{A}^{\mathrm{C}}$ and $\mathrm{B}^{\mathrm{C}}$ areneutrosophic open sets whose union is $X$. By (b), there exists neutrosophic feebly closed sets $F 1$ and $F 2$ such that $F 1 \leq A^{C}, F 2 \leq B^{C}$ and $F 1 V F 2=1_{N}$. Then $\mathrm{F}_{1}{ }^{\mathrm{C}}$ and $\mathrm{F}_{2}{ }^{\mathrm{C}}$ are disjoint neutrosophic feebly open sets containing $\mathrm{A}$ and $\mathrm{B}$ respectively. Therefore $\mathrm{X}$ is strongly neutrosophic feebly normal.

Theorem 4.5. Let $\mathrm{f}:\left(\mathrm{X}, \tau_{1}\right) \rightarrow\left(\mathrm{Y}, \tau_{2}\right)$ be a function.

- If $\mathrm{f}$ is injective, neutrosophic continuous, neutrosophic feebly open and $\mathrm{X}$ is strongly neutrosophic feebly normal then $\mathrm{Y}$ is strongly neutrosophic feebly normal.

- If $\mathrm{f}$ is neutrosophic feeblyirresolute, neutrosophic feebly closed and $\mathrm{Y}$ is strongly neutrosophic feebly normal then $\mathrm{X}$ is strongly neutrosophic feebly normal.

\section{Proof}

(a) Suppose X is strongly neutrosophic feebly normal. Let A and B be disjoint neutrosophic closed sets in Y. Since $\mathrm{f}$ is neutrosophic continuous, $\mathrm{f}^{-1}(\mathrm{~A})$ and $\mathrm{f}^{-1}(\mathrm{~B})$ are closed in $\mathrm{X}$. Since $\mathrm{X}$ is strongly neutrosophic feebly normal, there exist disjoint neutrosophic feebly open sets $U$ and Vin $X$ such that $f^{-1}(A) \leq U$ and $f^{-1}(B) \leq V$. Now $f^{-1}(A) \leq U \Rightarrow A \leq f(U)$ and $f^{-1}(B) \leq V \Rightarrow B \leq f(V)$. Since $f$ is a neutrosophic feebly open map, $f(U)$ and $f(V)$ are neutrosophic feebly open in $Y$. Also $\mathrm{U} \wedge \mathrm{V}=0_{N} \Rightarrow f(U \wedge V)=0_{N}$ and fis injective, then $f(U) \wedge f(V)=0_{N}$. Thus $f(U)$ and $f(V)$ are disjoint neutrosophic feebly open sets in $\mathrm{Y}$ containing $\mathrm{A}$ and $\mathrm{B}$ respectively. Thus, $\mathrm{Y}$ is strongly neutrosophic feebly normal.

(b)Suppose $\mathrm{Y}$ is neutrosophic feebly normal. Let A and B be disjoint neutrosophic closed sets in X. Since $\mathrm{f}$ is neutrosophic feebly irresolute and neutrosophic feebly closed, $\mathrm{f}(\mathrm{A})$ and $\mathrm{f}(\mathrm{B})$ are nSeutrosophic feebly closed in $\mathrm{Y}$. Since $\mathrm{Y}$ is neutrosophic feebly normal, there exist disjoint neutrosophic feebly open sets $U$ and $V$ in $Y$ such that $f(A) \leq U$ and $\mathrm{f}(\mathrm{B}) \leq \mathrm{V}$. That is $\mathrm{A} \leq \mathrm{f}^{-1}(\mathrm{U})$ and $\mathrm{B} \leq \mathrm{f}^{-1}(\mathrm{~V})$. Since $\mathrm{f}$ is neutrosophic feeblyirresolute, $\mathrm{f}^{-1}(\mathrm{U})$ and $\mathrm{f}^{-1}(\mathrm{~V})$ are disjoint neutrosophic feebly open such that $A \leq f^{-1}(U)$ and $B \leq f^{-1}(V)$. Thus $X$ is neutrosophic feebly normal.

\section{CONCLUSIONS}

In this paper, we have studiedneutrosophic feebly normal and strongly neutrosophic feebly normal spaces using neutrosophic feebly open andneutrosophic feebly closed. Also, found their relations among themselves and with already 
existing spaces. Also, we discussed some basic properties and the characterizations of already mentioned spaces.

\section{REFERENCES}

1. Albowi, SA, Salama, A, and Mohamed Eisa, 2013, New Concepts of Neutrosophic Sets, International Journal of Mathematics and Computer Applications Research, Vol.3(3), pp. 95-102.

2. Atanassov, K, 1986, Intuitionistic Fuzzy Sets, Fuzzy Sets and Systems, 20, pp. 87-96.

3. Bageerathi, K,JeyaPuvaneswari, P, 2019, Neutrosophic Feebly Connectedness And

4. Compactness(Submitted).

5. Chang, CL, 1968, Fuzzy Topological Spaces, J. Math. Anal. Appl. 24, pp.182-190.

6. Dogan Coker, 1997, An Introduction to Intuitionistic Fuzzy Topological Spaces, Fuzzy Sets and Systems, 88, pp. 81-89.

7. FloretinSmaradache, 2010, Neutrosophic Set:-A Generalization of Intuitionistic Fuzzy Set, Journal of Defense Resources Management. 1, pp. 107-116.

8. FloretinSmarandache, 2005,Neutrosophic set - A Generalization of the Intuitionistic Fuzzy Set, International Journal of Pure and Applied Mathematics, 24(3), pp. 287-297.

9. FloretinSmarandache, 2002, Neutrosophy and Neutrosophic Logic, First International Conference on Neutrosophy, Neutrosophic Logic, set, Probability, and statistics, University of New Mexico, Gallup, NM 87301. USA

10. FloretinSmarandache, 1999, A Unifying Field in Logics: Neutrosophic Logic,Neutrosophy, Neutrosophic Set, Neutrosophic Probability, American Research Press.

11. Murthy, S. N. Analysis \& Prediction Of Mischievous Behavior Of Vehicle Using Anpr And Dbscan.

12. Iswarya, P, and Bageerathi, K, 2016, On Neutrosophic Semi-Open Sets in Neutrosophic Topological Spaces, International Journal of Mathematics Trends and Technology (IJMTT),Vol 37(3), pp. 24-33.

13. JeyaPuvaneswari, P, Bageerathi, K, 2017, On Neutrosophic Feebly open sets in Neutrosophic topological spaces, International Journal of Mathematics Trends and Technology, Vol. 41(3), pp. 230 - 237.

14. JeyaPuvaneswari, P, Bageerathi, K, 2019, On Neutrosophic Feebly Continuous Functions Journal of Emerging Technologies and Innovative Research, Vol. 6 (2) (ISSN No. 2349 - 5162) (UGC Approved Journal No. 63975)

15. JeyaPuvaneswari, P, Bageerathi, K, 2019, Some Functions Concerning Neutrosophic Feebly Open \& Closed Sets. (Submitted).

16. Parimala .M, Jeevitha .R, Jafari. S, Smarandache .F, Neutrosophic $\alpha \Psi$-omeomorphism in Neutrosophic Topological Spaces, Information Journal, 2018,9,187.

17. Salama, AA, Smarandache, Floretin, and Valeri, K, 2014,Neutrosophic Closed Set and Neutrosophic Continuous Functions, Neutrosophic Sets and Systems, 4, pp. 4-8.

18. Salama, AA, and Alblowi, SA, 2012, Generalized Neutrosophic Set and Generalized Neutrousophic Topological Spaces, Journal computer Sci. Engineering, Vol. (2) No. 7.

19. Salama, AA, Alblowi, AA, 2012, Neutrosophic Set and Neutrosophic Topological

20. Space, ISOR Journal of Mathematics, Vol. (3), Issue (4), pp. 31-35. 
21. Alblowi, S. A., Salama, A. A., \& Eisa, M. (2013). New concepts of neutrosophic sets. International Journal of Mathematics and Computer Applications Research (IJMCAR), 3(4), 95-102.

22. Turnali, $N$ and Coker,D, 2000, Fuzzy connectedness in intuitionistic fuzzy topological spaces, Fuzzy Sets and Systems, 116, 369-375.

23. Zadeh, LA, 1965, Fuzzy Sets, Inform and Control, Vol. 8, pp. 315-353. 
